\title{
Optimal Decision for Fashion Supply Chains with Service Level Constraint and Controllable Lead Time
}

\author{
Guo Li, ${ }^{1,2}$ Yu-chen Kang, ${ }^{1,2}$ and Guan $\mathrm{Xu}^{3}$ \\ ${ }^{1}$ School of Management and Economics, Beijing Institute of Technology, Beijing 100081, China \\ ${ }^{2}$ Center for Energy and Environmental Policy Research, Beijing Institute of Technology, Beijing 100081, China \\ ${ }^{3}$ Economic and Management School, Wuhan University, Wuhan 410081, China \\ Correspondence should be addressed to Guan Xu; dageguan@gmail.com
}

Received 4 June 2014; Accepted 10 July 2014

Academic Editor: Tsan-Ming Choi

Copyright (C) 2015 Guo Li et al. This is an open access article distributed under the Creative Commons Attribution License, which permits unrestricted use, distribution, and reproduction in any medium, provided the original work is properly cited.

\begin{abstract}
We study a two-echelon supply chain inventory model with controllable lead time and service level constraint in fashion supply chains, in which we assume that the unit cost of compressing lead time follows exponential distribution. Under these conditions we investigate the optimal ordering quantity and production quantity in the fashion supply chain by minimizing the joint total cost. Simultaneously, we work out the boundaries of ordering quantity and production quantity, which simplify the computation. Furthermore, numerical examples are presented to test the feasibility of the model. The results show that assuming the unit cost of compressing lead time in accordance with exponential distribution is realistic. It also notices that the optimal order and production decision for fashion supply chains are constrained obviously by the service level and safety factors. What is more, the holding cost rate of both the service level and safety factors has a certain influence on it. And by further analyzing on some references in the last part, we have done some extensions and found some interesting results.
\end{abstract}

\section{Introduction}

The fashion industry has some specific features like short product life cycle, volatile, unpredictable demand, and tremendous product variety. Therefore, an efficient, flexible, and complex supply chain is in urgent need to match those features [1]. Efforts have been made in optimal decision for supply chains with demand variations, credit period, and price discount [2] and also in production and ordering decision in supply chains with uncertainty in two-echelon yields and demand. Even dual-sourcing supply chain model is considered to deal with uncertain demand or supply disruption and other potential problems in supply chains [35]. But for the fashion industry, the service quality also should be considered.

So this paper focuses on efficiency, which requires higher speed. And the higher speed will be generated mainly from shorter lead time with service level constraint. Therefore, we add to these growing efforts of optimizing fashion supply chains by analyzing the order and production decisions under service level constraint and controllable lead time.

To the best of our knowledge, the issue of fashion supply chains' optimal decision under service level constraint and controllable lead time, with the assumption that the cost of compressing lead time is to be exponentially distributed, has never been adequately investigated. The optimal backorder decision and lost sales problem have a close bearing with the service level constraint and controllable lead time [6]. Speed is becoming a strategic competitive weapon and purchasing optimal lead time gradually appeals to the enterprises' and researchers' attention. So it is of great significance to study the order and production decisions of the fashion supply chain with service level constraint and controllable lead time. This paper mainly studies the ordering, production quantity in the fashion supply chain with service level constraint and controllable lead time. It analyzes how to determine the proper point of order quantity and production quantity so as to reach the minimum cost and lead time with service level 
constraint. To address these problems, the paper sets out to answer the following questions.

(1) How are the inventory models when considering the cost function of compressing lead time following exponential distribution and its impact on the expected total cost?

(2) With the service level constraint and controllable lead time, what is the optimal decision for fashion supply chains, and how will it affect the optimal decision for fashion supply chains with safety factor?

(3) How should each party look for the optimal order quantity and production quantity in fashion supply chains?

The remainder of the paper is organized as follows. Section 2 contains a review of relevant literature. The model assumptions of fashion supply chains are described in Section 3. The buyer's and vendor's inventory model, joint model with controllable lead time are given in Section 4 . In Section 5, solution technique steps are shown in detail. To gain thorough comprehension of our dedicated policy, numerical computations and parametric analyses are conducted in Section 6. Extensions are shown in Section 7. Then conclusions and suggestions are made for further researches in the last section.

\section{Literature Review}

In the era where efficiency is predominantly seen as the priority, pursuing efficient model of supply chain becomes people's goal with consideration of controllable lead time. Here the efficiency does not simply mean to shorten distance and to improve the speed of the car; it also includes compressing the lead time. Shortening lead time can improve customer service levels and reduce stock costs, ensure safety stock, and so on. As a result, lead time has attracted many scholars' attention, and numerous inventory models have taken lead time and service level into consideration [7-13]. Now we review related literatures from the following two aspects: lead time and service level.

In the past, various models and mechanisms have been developed to provide a more effective and scientific way for compressing the controllable lead time. Liao and Shyu [14] are among the first ones who assume that lead time is negotiable and can be decomposed into several components, each having a different piecewise linear crash cost function for lead time reduction. After that more and more inventory models concerning lead time reduction issue have been developed. For example, Ben-Daya and Raouf [7] extend Liao and Shyu's model by viewing both lead time and order quantity as decision variables and propose management and control strategy for the lead time on account of those variables. Based on this, Ouyang et al. [15] further consider the circumstances of backorders and stock-out. Moon and Choi [16] and Lan et al. [17] find individual optimal order quantity and optimal lead time for a mixed inventory model, and they develop a simplified solution procedure under a continuous inventory system. Ouyang et al. [18] attempt to derive a continuous review inventory model with defective items, where the order quantity, reorder point, and lead time are taken as decision variables. First they assume that the lead time demand follows a normal distribution and then loosen this assumption by only assuming that the mean and variance of lead time demand are known. Then Ouyang and $\mathrm{Wu}$ [19] for the first time take the distribution-free condition into inventory model. They loosen the assumption of the cumulative lead time distribution demand while at the same time develop the minimized distribution-free procedure to determine the optimal lead time and optimal order quantity. With that Ouyang et al. [20] investigate the impact of investing in quality improvement and lead time reduction on the integrated vendor-buyer inventory model with partial backorders, and the minimized distribution-free procedure is applied to solve this problem. On this basis, Jha and Shanker [21] extend the integrated vendor-buyer inventory model to single-vendor multi-buyers integrated production-inventory model. In 2013, there is a big breakthrough on the cost function of compressing lead time. Li et al. [22] for the first time propose that the unit cost of compressing lead time in most periods includes not only the fixed part, but also the variable parts and that these variable parts are sensitive to order quantity. In the real life, more reasonable lead time unit compressing cost should be segmented and be sensitive to order quantity and productivity [23-25].

But, for this paper, considering the characteristics of fashion supply chains, we assume that the unit cost of compressing lead time is exponentially distributed. That is because as we all know, in the stage of the clothing of productive processing, the longer the lead time is, the lower the unit cost of compressing lead time is correspondingly. So the unit lead time compressing cost should be shorter during this lead time. There are two reasons why we model stochastic lead time to follow an exponential distribution. First, it is reasonable to assume that a shipment process/system stays as good as new until the shipment finally reaches the destination; that is, the operation of the shipment system has no aging process over the lead time. To fulfill this property, the exponential distribution is the only one to choose among continuous distributions. Second, the exponential distribution is widely used to model many time-oriented variables, such as waiting time or lead time between occurrences of events and lifetime of electrical, mechanical devices or the fashion industry [26].

Most above-mentioned literatures bring the theory of controllable lead time into the inventory model, but few of them ever consider the service level constraint simultaneously. As for the service level, now the factor has also been often introduced into the inventory model, because it has come to the decision makers that lack of this factor will cause stock-out, customer loss, and so on, of course, which is especially significant for fashion supply chains. Ouyang and $\mathrm{Wu}$ [8] suggest that, instead of having a stock-out term in the objective function, a service level constraint, which implies that the stock-out level per cycle is bounded, is added to the model. Chiu et al. [27] consider a market with respect to the service level decision on a particular common service product, finding that one service provider chooses to deviate 
from the equilibrium service level with the goal of improving market share. So the factor is necessary when considering the stock-out problem with controllable lead time, but few of the previous literatures have included the factor of service level constraint.

Therefore, in analyzing the supply chains of fashion industry, this paper takes the service level into account and also considers the condition that the cost part of compressing controllable lead time is exponentially distributed.

\section{Assumptions}

In the analysis, the major presumptions are as follows.

(1) There is a single-vendor and a single-buyer and they deal with a single item with the buyer using a continuous review inventory policy and whenever inventory level falls below the reorder point $r$, the order is placed.

(2) The reorder point is determined by $r=\mu L+k \sigma \sqrt{L}$, where $k$ is safety factor. The mean value of vendor's demand per unit time is $\mu$, and the variance is $\sigma^{2}$.

(3) Because the buyer is allowed to be out of stock, its expectations of stock during the order cycle $B(r)=$ $E(X-r)^{+},(X-r)^{+}=\max (X-r, 0)$.

(4) According to the definition of service level, $E\left([X-r]^{+}\right) / q \leq \theta$.

(5) The lead-time-dependent cost follows a function, which is assumed to be exponentially distributed with parameters $a$ and $b$. Mathematically, the cost of compressing lead time is

$$
C_{L}=a e^{-b L}
$$

in which $L \geq 0, a \geq 0$, and $b \geq 0$.

\section{Model Formulation}

In this paper, order quantity, production quantity, and lead time are variable. In order to look for the optimal order quantity, optimal production quantity, and optimal lead time with service level constraint, we establish a model for the total expected cost of the inventory system and minimize it to attain the optimal value of the decisive variables.

4.1. The Buyer's Inventory Model (Without Price Discount). In this paper, for the buyer's expected total cost per unit time, we consider it is the sum of purchasing cost, holding cost, shortage cost, and the lead time crash cost. The formula is as follows:

$$
C_{\mathrm{BT}}=C_{\mathrm{BP}}+C_{\mathrm{BH}}+C_{\mathrm{BS}}+C_{\mathrm{BL}}
$$

The buyer's expected purchasing cost per unit time is

$$
C_{\mathrm{BP}}=P_{v} \mu .
$$

For the holding cost per unit time, it contains order quantity, lost sales quantity, and backorder quantity. Hence, the holding quantity per unit time is

$$
\begin{aligned}
Q_{\mathrm{BH}} \approx \frac{1}{2}[ & q+E(r-X)^{+}+(1-\beta) B(r)+E(r-X)^{+} \\
& +(1-\beta) B(r)] .
\end{aligned}
$$

According to Assumption 5 in the paper of Hadley and Whitin [28], we can transform (4) into the following equation and also know that $\psi(k)=\left(\sqrt{1+k^{2}}-k\right) / 2$ :

$$
\mathrm{Q}_{\mathrm{BH}}=\frac{q}{2}+k \sigma \sqrt{L}+(1-\beta) \sigma \sqrt{L} \psi(k) .
$$

Hence, the holding cost per unit time is

$$
C_{\mathrm{BH}}=r_{b} P_{v}\left\{\frac{q}{2}+k \sigma \sqrt{L}+(1-\beta) \sigma \sqrt{L} \psi(k)\right\} .
$$

With regard to the shortage cost, we assume that a price discount of unit reorder product is offered to the customers who are willing to wait for the next replenishment. So the shortage cost contains discount cost and the cost of losing orders, which is shown by the following formula:

$$
\begin{aligned}
C_{\mathrm{BS}} & =\frac{\mu}{q} P B(r)\left[(1-\beta)+\left(1-\alpha^{\prime}\right) \beta\right] \\
& =\frac{\mu}{q} P \sigma \sqrt{L} \psi(k)\left(1-\alpha^{\prime} \beta\right) .
\end{aligned}
$$

Finally, the buyer's expected total cost per unit time is

$$
\begin{aligned}
C_{\mathrm{BT}}= & P_{v} \mu+r_{b} P_{v}\left\{\frac{q}{2}+k \sigma \sqrt{L}+(1-\beta) \sigma \sqrt{L} \psi(k)\right\} \\
& +\frac{\mu}{q} P \sigma \sqrt{L} \psi(k)\left(1-\alpha^{\prime} \beta\right)+\frac{\mu}{q} C_{L} \\
= & P_{v} \mu+r_{b} P_{v}\left(\frac{q}{2}+k \sigma \sqrt{L}\right) \\
& +\sigma \sqrt{L} \psi(k)\left[r_{b} P_{v}(1-\beta)+\frac{P \mu}{q}\left(1-\alpha^{\prime} \beta\right)\right]+\frac{\mu}{q} C_{L} .
\end{aligned}
$$

4.2. The Vendor's Inventory Model. Similar to most of the literatures dealing with controllable lead time with the same setting for the vendor (e.g., Ouyang et al., 2004 [23]), the vendor's expected cost per unit time is represented as

$$
\begin{aligned}
C_{\mathrm{VT}} & =\frac{S \mu}{m q}+\frac{r_{v} P_{v} q}{2}\left[m\left(1-\frac{\mu}{P_{c}}\right)-1+\frac{2 \mu}{P_{c}}\right] \\
& =\frac{S \mu}{m q}+\frac{r_{v} P_{v} q(m-1)}{2}+\frac{(2-m) \mu r_{v} P_{v} q}{2 P_{c}} .
\end{aligned}
$$


Then the joint expected cost of the inventory system can be obtained as follows:

$$
\begin{aligned}
C_{\mathrm{JT}}= & P_{v} \mu+r_{b} P_{v}\left(\frac{q}{2}+k \sigma \sqrt{L}\right) \\
& +\sigma \sqrt{L} \psi(k)\left[r_{b} P_{v}(1-\beta)+\frac{P \mu}{q}\left(1-\alpha^{\prime} \beta\right)\right]+\frac{\mu}{q} C_{L} \\
& +\frac{S \mu}{m q}+\frac{r_{v} P_{v} q(m-1)}{2}+\frac{(2-m) \mu r_{v} P_{v} q}{2 P_{c}} \\
& \text { s.t. } \frac{E\left([X-r]^{+}\right)}{q} \leq \theta \text { equal to } \frac{\sigma \sqrt{L} \psi(k)}{q} \leq \theta .
\end{aligned}
$$

\section{Solution Technique}

In order to obtain the optimal order quantity, optimal production quantity, and optimal lead time with service level constraint, we minimize the joint cost function and calculate the first derivative and the second derivative of all the variables.

5.1. Model Analysis. Calculate the derivative $C_{\mathrm{JT}}$ on $q$ and $m$, respectively. That is,

$$
\begin{aligned}
\frac{\partial C_{\mathrm{JT}}}{\partial q}= & \frac{r_{b} P_{v}}{2}-\frac{\mu}{q^{2}}\left[C_{L}+P\left(1-\alpha^{\prime} \beta\right) \sigma \sqrt{L} \psi(k)+\frac{S}{m}\right] \\
& +\frac{r_{v} P_{v}(m-1)}{2}+\frac{(2-m) \mu r_{v} P_{v}}{2 P_{c}} \\
\frac{\partial^{2} C_{\mathrm{JT}}}{\partial q^{2}}= & 2 \frac{\mu}{q^{3}}\left[C_{L}+P\left(1-\alpha^{\prime} \beta\right) \sigma \sqrt{L} \psi(k)+\frac{S}{m}\right]>0 .
\end{aligned}
$$

Then we know $\partial^{2} C_{\mathrm{JT}} / \partial q^{2}>0$, which means that $C_{\mathrm{JT}}$ are convex in each value of $q$ when $q \geq 0$. So let (11) equal zero; we can get

$$
q_{\mathrm{JT}}=\left[\frac{2 \mu\left[C_{L}+P\left(1-\alpha^{\prime} \beta\right) \sigma \sqrt{L} \psi(k)+(S / m)\right]}{r_{b} P_{v}+r_{v} P_{v}\left[m-1+\left((2-m) \mu / P_{c}\right)\right]}\right]^{1 / 2} .
$$

The derivative of $m$ is

$$
\begin{gathered}
\frac{\partial C_{\mathrm{JT}}}{\partial m}=-\frac{S \mu}{m^{2} q}+\frac{r_{v} P_{v} q}{2}-\frac{\mu r_{v} P_{v} q}{2 P_{c}} \\
\frac{\partial^{2} C_{\mathrm{JT}}}{\partial m^{2}}=\frac{2 S \mu}{m^{3} q}>0 ;
\end{gathered}
$$

that is,

$$
\frac{\partial^{2} C_{\mathrm{JT}}}{\partial m^{2}}>0
$$

According to (15) and (12), obviously the function is convex in each $m$ and $q$ for $C_{\mathrm{JT}}$; therefore, we can obtain the following corollaries.
Corollary 1. The function of $C_{J T}(q, L, m)$ is joint convex in $(q, m)$ when $L \in\left[L_{i}, L_{i-1}\right]$ is fixed.

Corollary 2. With service level constraint the optimal order quantity is equal to $\max \left(q_{J T}, \sigma \sqrt{L} \psi(k) / \theta\right)$ and $q_{\min } \leq q_{J T} \leq$ $q_{\max }$

Proof. Because service level $\sigma \sqrt{L} \psi(k) / q \leq \theta$, we can know $\sigma \sqrt{L} \psi(k) / \theta \leq q$. When $q_{\mathrm{JT}} \geq \sigma \sqrt{L} \psi(k) / \theta$, the optimal order quantity is $q_{\mathrm{JT}}$. But if $q_{\mathrm{JT}}<\sigma \sqrt{L} \psi(k) / \theta$, the optimal order quantity is $\sigma \sqrt{L} \psi(k) / \theta$.

When the lead time is not compressed or is compressed to the minimum, we can obtain that $0 \leq a e^{-b L} \leq a$. And then substitute this formula into (13), there will be

$$
\begin{aligned}
& {\left[\frac{2 \mu\left[P\left(1-\alpha^{\prime} \beta\right) \sigma \sqrt{L} \psi(k)+(S / m)\right]}{r_{b} P_{v}+r_{v} P_{v}\left[m-1+\left((2-m) \mu / P_{c}\right)\right]}\right]^{1 / 2}} \\
& \leq q_{\mathrm{JT}} \leq\left[\frac{2 \mu\left[a+P\left(1-\alpha^{\prime} \beta\right) \sigma \sqrt{L} \psi(k)+(S / m)\right]}{r_{b} P_{v}+r_{v} P_{v}\left[m-1+\left((2-m) \mu / P_{c}\right)\right]}\right]^{1 / 2} .
\end{aligned}
$$

Then, $q_{\min } \leq q_{\mathrm{JT}} \leq q_{\max }$.

5.2. Optimal Production Quantity Decision. First, we consider the case when $q_{\mathrm{JT}}$ is not constrained by service level; that is to say, $q_{\mathrm{JT}} \geq \sigma \sqrt{L} \psi(k) / \theta$. Since $\partial^{2} C_{\mathrm{JT}} / \partial m^{2}=2 S \mu / m^{3} q>0$, then $C_{\mathrm{JT}}(q, L, m)$ is convex in $m$ for fixed $q$ and $L \in\left[L_{i}, L_{i-1}\right]$, which implies that there must be an optimal $m=m_{\mathrm{JT}}$ to meet the following formula:

$$
\begin{aligned}
& C_{\mathrm{JT}}\left(m_{\mathrm{JT}}\right) \leq C_{\mathrm{JT}}\left(m_{\mathrm{JT}}-1\right) \\
& C_{\mathrm{JT}}\left(m_{\mathrm{JT}}\right) \leq C_{\mathrm{JT}}\left(m_{\mathrm{JT}}+1\right) .
\end{aligned}
$$

First, substitute (13) into (10), and then to facilitate the calculation we choose to ignore the terms of $C_{\mathrm{JT}}\left(m_{\mathrm{JT}}\right)$ that are independent of $m$, so we have

$$
\begin{aligned}
F(m)=\{ & {\left[C_{L}+P\left(1-\alpha^{\prime} \beta\right) \sigma \sqrt{L} \psi(k)+\frac{S}{m}\right] } \\
& \left.\times\left[r_{b} P_{v}+r_{v} P_{v}\left(m-1+\frac{(2-m) \mu}{P_{c}}\right)\right]\right\}^{1 / 2} .
\end{aligned}
$$

So,

$$
\begin{aligned}
F^{2}(m)= & {\left[C_{L}+P\left(1-\alpha^{\prime} \beta\right) \sigma \sqrt{L} \psi(k)+\frac{S}{m}\right] } \\
& \times\left[r_{b} P_{v}+r_{v} P_{v}\left(m-1+\frac{(2-m) \mu}{P_{c}}\right)\right] .
\end{aligned}
$$

Ignore the terms of $F^{2}(m)$ without $m$, and let

$$
\begin{aligned}
G(m)= & r_{v} m\left[C_{L}+P\left(1-\alpha^{\prime} \beta\right) \sigma \sqrt{L} \psi(k)\right]\left(1-\frac{\mu}{P_{c}}\right) \\
& +\frac{S}{m}\left(r_{b}-r_{v}+\frac{2 \mu r_{v}}{P_{c}}\right) .
\end{aligned}
$$


Then we have

$$
\begin{aligned}
& G\left(m_{\mathrm{JT}}\right) \leq G\left(m_{\mathrm{JT}}-1\right) \\
& G\left(m_{\mathrm{JT}}\right) \leq G\left(m_{\mathrm{JT}}+1\right) .
\end{aligned}
$$

By calculating the above formula, we can obtain

$$
\begin{aligned}
m_{\mathrm{JT}} & \left(m_{\mathrm{JT}}-1\right) \\
& \leq \frac{S\left(r_{b}-r_{v}+\left(2 \mu r_{v} / P_{c}\right)\right)}{r_{v}\left[C_{L}+P\left(1-\alpha^{\prime} \beta\right) \sigma \sqrt{L} \psi(k)\right]\left(1-\left(\mu / P_{c}\right)\right)} \\
& \leq m_{\mathrm{JT}}\left(m_{\mathrm{JT}}+1\right) .
\end{aligned}
$$

Second, if we consider $q_{\mathrm{JT}}$ with service level constraint, that means $q_{\mathrm{JT}} \leq \sigma \sqrt{L} \psi(k) / \theta$ and the optimal order quantity is $\sigma \sqrt{L} \psi(k) / \theta$.

In this process, ignore the part $C_{\mathrm{BT}}$ without $m$; let

$$
\begin{aligned}
& C_{\mathrm{VT}}\left(m_{\mathrm{JT}}\right) \leq C_{\mathrm{VT}}\left(m_{\mathrm{JT}}-1\right) \\
& C_{\mathrm{VT}}\left(m_{\mathrm{JT}}\right) \leq C_{\mathrm{VT}}\left(m_{\mathrm{JT}}+1\right) ;
\end{aligned}
$$

then we have

$$
\begin{aligned}
& \frac{S \mu}{m_{\mathrm{JT}} q}+\frac{r_{v} P_{v} q\left(m_{\mathrm{JT}}-1\right)}{2}+\frac{\left(2-m_{\mathrm{JT}}\right) \mu r_{v} P_{v} q}{2 P_{c}} \\
& \leq \frac{S \mu}{\left(m_{\mathrm{JT}}-1\right) q}+\frac{r_{v} P_{v} q\left(m_{\mathrm{JT}}-2\right)}{2}+\frac{\left(3-m_{\mathrm{JT}}\right) \mu r_{v} P_{v} q}{2 P_{c}} \\
& \frac{S \mu}{m_{\mathrm{JT}} q}+\frac{r_{v} P_{v} q\left(m_{\mathrm{JT}}-1\right)}{2}+\frac{\left(2-m_{\mathrm{JT}}\right) \mu r_{v} P_{v} q}{2 P_{c}} \\
& \leq \frac{S \mu}{\left(m_{\mathrm{JT}}+1\right) q}+\frac{r_{v} P_{v} q m_{\mathrm{JT}}}{2}+\frac{\left(1-m_{\mathrm{JT}}\right) \mu r_{v} P_{v} q}{2 P_{c}} .
\end{aligned}
$$

Simplify it and there will be

$$
m_{\mathrm{JT}}\left(m_{\mathrm{JT}}-1\right) \leq \frac{2 S \mu}{r_{v} P_{v} q^{2}\left(1-\left(\mu / P_{c}\right)\right)} \leq m_{\mathrm{JT}}\left(m_{\mathrm{JT}}+1\right) .
$$

Substitute $q_{\mathrm{TT}}=\sigma \sqrt{L} \psi(k) / \theta$ into (26); then

$$
\begin{aligned}
m_{\mathrm{JT}}\left(m_{\mathrm{JT}}-1\right) & \leq \frac{2 S \mu \theta^{2}}{r_{v} P_{v} \sigma^{2} L \psi^{2}(k)\left(1-\left(\mu / P_{c}\right)\right)} \\
& \leq m_{\mathrm{JT}}\left(m_{\mathrm{JT}}+1\right) .
\end{aligned}
$$

With (22) and (26) we can estimate the range of $m$, where $0 \leq a e^{-b L} \leq a$. And that will help working out the minimum joint cost and the optimal order quantity, optimal production quantity, and optimal lead time with service level constraint.

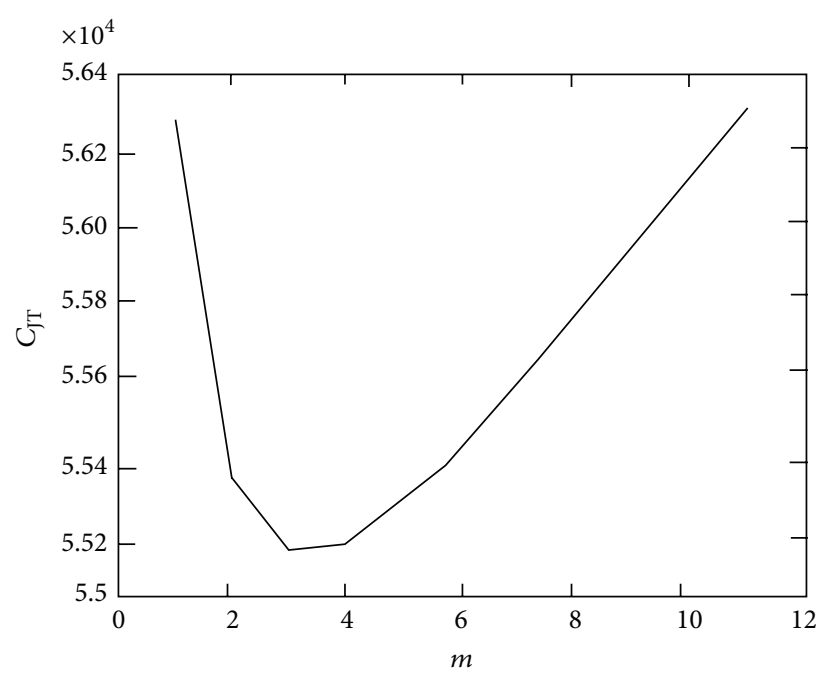

FIGURE 1: Variance of the optimal joint total cost under different values of $m$.

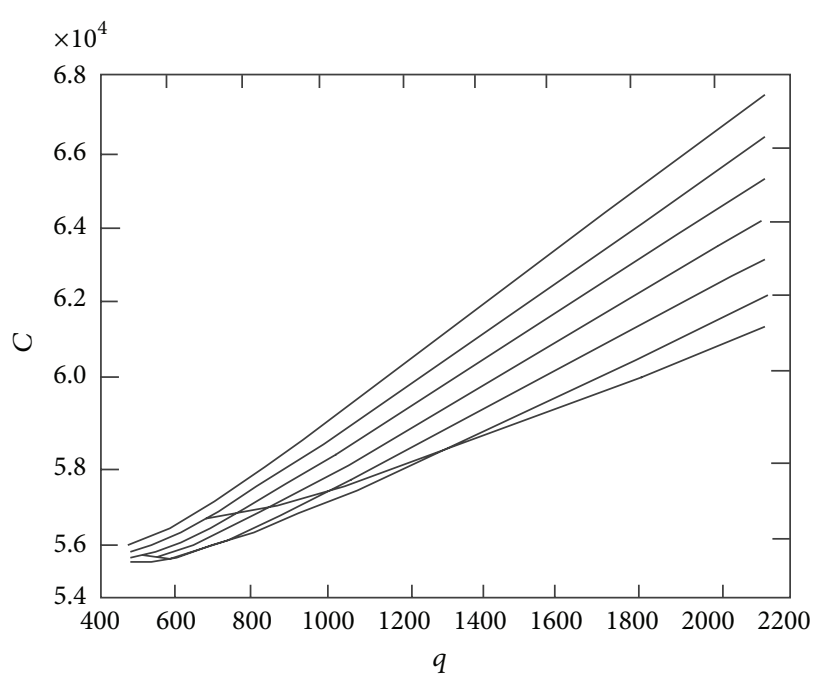

Figure 2: The influence of $q$ on the optimal joint total cost under different values of $m$.

\section{Numerical Analysis}

In order to test the feasibility of the model, we consider an inventory system with the following characteristics: $\theta=1 \%$, $P_{v}=30$ USD/unit, $P=100 \mathrm{USD} /$ unit, $r_{b}=0.15, r_{v}=0.2$, $P_{c}=2000$ units $/ \mathrm{a}, \beta=0.5, \alpha^{\prime}=0.8, a=1000, b=3, S=$ $1000 \mathrm{USD} /$ time, $\sigma=15$ units/week, $\mu=1650$ units/a, and $k=0.1$.

Figures 1 and 2 once again prove that (10) and (15) are correct in reality. This shows that $C_{\mathrm{TT}}$ are convex in each $m$ and $q$, which is corresponding with Corollary 1 . In Figure 1, the optimal joint total inventory cost decreases until $m$ reaches a certain value, and then it begins to increase. That means the vendor is up to the scale, so there is an optimal $m$ making the joint total inventory cost minimum.

Table 1 shows the optimal order quantity, lead time, production quantity, and the minimum cost with different 
TABLE 1: Computed results for different values of $\theta$ and $k$ (lead time in weeks).

\begin{tabular}{cccccc}
\hline$\theta$ & $k$ & $m^{*}$ & $L^{*}$ & $q^{*}$ & $C_{\mathrm{JT}}^{*}$ \\
\hline \multirow{4}{*}{0.015} & 0 & 3 & 1.1 & 347 & 48778 \\
& 0.5 & 4 & 1.3 & 258 & 48324 \\
& 1.5 & 5 & 1.6 & 194 & 47822 \\
& 4.5 & 8 & 1.8 & 116 & 47427 \\
& 9.5 & 10 & 1.9 & 90 & 47401 \\
\hline \multirow{4}{*}{0.005} & 0 & 2 & 0.6 & 542 & 49313 \\
& 0.5 & 2 & 1 & 441 & 48626 \\
& 1.5 & 4 & 1.3 & 242 & 47884 \\
& 4.5 & 8 & 1.8 & 116 & 47427 \\
0.001 & 9.5 & 10 & 1.9 & 90 & 47401 \\
\hline \multirow{4}{*}{0} & 0 & 2 & 0.1 & 1107 & 51186 \\
& 0.5 & 2 & 0.1 & 684 & 50751 \\
& 1.5 & 2 & 0.3 & 580 & 49732 \\
& 4.5 & 2 & 1.1 & 403 & 48237 \\
\hline
\end{tabular}

TABLE 2: Optimal results for different values of $r_{b}$ and $r_{v}$ (lead time in weeks).

\begin{tabular}{lccccc}
\hline$r_{b}$ & $r_{v}$ & $m^{*}$ & $L^{*}$ & $q^{*}$ & $C_{\mathrm{JT}}^{*}$ \\
\hline \multirow{3}{*}{0.10} & 0.10 & 4 & 0.8 & 607 & 53569 \\
& 0.15 & 4 & 0.8 & 578 & 53514 \\
& 0.20 & 4 & 0.9 & 583 & 53461 \\
\hline \multirow{3}{*}{0.15} & 0.10 & 4 & 0.7 & 568 & 54161 \\
& 0.15 & 4 & 0.7 & 540 & 54093 \\
& 0.20 & 4 & 0.8 & 550 & 54024 \\
\hline \multirow{3}{*}{0.20} & 0.10 & 3 & 0.7 & 568 & 54680 \\
& 0.15 & 3 & 0.7 & 540 & 54611 \\
& 0.20 & 3 & 0.8 & 550 & 54542 \\
\hline
\end{tabular}

service level and safety factor. This table verifies that, with safety factor increasing, the order quantity decreases and the joint cost increases. We also know that if the retailer has high request for the level of service, he/she should burden higher cost accordingly. When $\theta$ is low enough, the optimal joint cost is much higher than the cost with higher $\theta$ and it changes quickly with variance of the safety factor. Whatever the value of $\theta$ is, the influence of $k$ on the optimal inventory joint total cost gradually becomes greater until it reaches a certain value, and then its influence becomes weak with $k$ increasing.

From Table 2, we can see the effect of holding costs of both the vendor and the buyer on $C_{\mathrm{TT}}$. Since $r_{b}$ of a buyer and $r_{v}$ of a vendor may not be the same in practice, we analyze the effect of holding cost rate on the joint total expected cost as shown in Table 2. When $r_{b}>r_{v}$, as we can see, the buyer tends to order a small quantity, but that has small influence on the vendor. It indicates that when the buyer has a higher holding cost rate, he/she is more likely to order a smaller quantity each time and to keep his/her average inventory level as low as possible by maintaining higher safety stock. When $r_{b}$ reaches a certain value, it will have less and less influence on his/her order quantity. But when $r_{b}<r_{v}$, the buyer will order a larger quantity and the vendor changes little. That means if the buyer has a smaller holding cost rate, he/she is more likely to order a larger quantity each time to keep his/her average inventory level, but small changes in $r_{v}$ have little effect on product quantity for the vendor.

\section{Extensions}

By reviewing the previous researches, we also find some other interesting issues. The two extensions are shown in detail as follows.

7.1. Price Discount Incentives. Does the optimal decision we have researched above make the fashion supply chain reach its optimality? Is there any other approach to lower their costs? With questions of this kind in mind, we pay attention to the incentive way of offering price discount to the retailer $[29,30]$. But how does the supplier determine the specific discount value to make both parties in the chain decrease their cost? Then we calculate the boundary of the price discount by the following analysis, which should ensure no damage to both sides and reduce the cost of the entire fashion supply chain.

If the vendor offers the following price discount scheme,

$$
P_{v}^{\prime}= \begin{cases}P_{v}, & q<q^{*}, L \neq L^{*} \\ (1-\alpha) P_{v}, & q \geq q^{*}, L=L^{*}\end{cases}
$$

as an encouragement for the buyer to make decisions to maximize the expected profit and minimize the expected cost of the entire supply chain, then the buyer's cost will be

$$
\begin{aligned}
C_{\mathrm{BT}}^{\prime}= & (1-\alpha) P_{v} \mu+r_{b} P_{v}(1-\alpha)\left(\frac{q^{*}}{2}+k \sigma \sqrt{L^{*}}\right) \\
& +\theta \sqrt{L^{*}} \psi(k)\left[r_{b} P_{v}(1-\alpha)(1-\beta)+P \mu\left(1-\alpha^{\prime} \beta\right)\right] \\
& +\frac{\mu}{q^{*}} C_{L} .
\end{aligned}
$$

The buyer accepts the price discount scheme only if the price compensation that the buyer gets exceeds its increased cost, which means

$$
\alpha P_{v} \mu \geq C_{\mathrm{BT}}^{\prime}-C_{\mathrm{BT}} \Longrightarrow \alpha \geq \frac{C_{\mathrm{BT}}^{\prime}-C_{\mathrm{BT}}}{P_{v} \mu} .
$$

Similarly, the vendor's cost is

$$
C_{\mathrm{VT}}^{\prime}=\frac{S \mu}{m q^{*}}+\frac{r_{v} P_{v}(1-\alpha) q^{*}}{2}\left[m\left(1-\frac{\mu}{P_{c}}\right)-1+\frac{2 \mu}{P_{c}}\right] \text {. }
$$

The vendor accepts the price discount scheme only if the price compensation that the vendor gives to the buyer is less than his/her decreased cost; that is,

$$
\alpha P_{v} \mu \leq C_{\mathrm{VT}}-C_{\mathrm{VT}}^{\prime} \Longrightarrow \alpha \leq \frac{C_{\mathrm{VT}}-C_{\mathrm{VT}}^{\prime}}{P_{v} \mu} .
$$

Based on the above discussion we can obtain the result as follows. 
Corollary 3. The vendor and the buyer will accept the price discount mechanism, if

$$
\frac{C_{B T}^{\prime}-C_{B T}}{P_{v} \mu} \leq \alpha \leq \frac{C_{V T}-C_{V T}^{\prime}}{P_{\nu} \mu} .
$$

Both of their expected costs will be decreased if the proper price discount meeting the above conditions really exists.

7.2. Lead Time Sensitive to Productivity. By instinct we know that lead time will be compressed shorter with productivity increasing. Is that absolutely right? If it is not right, is there an optimal productivity? To validate these questions, according to Assumption 6 of [22], we assume that controllable lead time is sensitive to order quantity and productivity, which is

$$
C_{L}^{\prime}=\left(a_{i}+b_{i} \frac{q}{P_{c}}\right)\left(L_{i-1}-L\right)+\sum_{j=1}^{i-1}\left(a_{j}+b_{j} \frac{q}{P_{c}}\right)\left(T_{j}-t_{j}\right)
$$

in which $L \in\left[L_{i}, L_{i-1}\right]$ and $j=1,2, \ldots, i$.

So

$$
\begin{aligned}
C_{\mathrm{JT}}^{\prime}= & P_{v} \mu+r_{b} P_{v}\left(\frac{q}{2}+k \sigma \sqrt{L}\right) \\
& +\sigma \sqrt{L} \psi(k)\left[r_{b} P_{v}(1-\beta)+\frac{P \mu}{q}\left(1-\alpha^{\prime} \beta\right)\right]+\frac{\mu}{q} C_{L}^{\prime} \\
& +\frac{S \mu}{m q}+\frac{r_{v} P_{v} q(m-1)}{2}+\frac{(2-m) \mu r_{v} P_{v} q}{2 P_{c}} .
\end{aligned}
$$

We take a derivative of (34) with respect to $P_{c}$, and let $C_{L b}=b_{i}\left(L_{i-1}-L\right)+\sum_{j=1}^{i-1} b_{j}\left(T_{j}-t_{j}\right)$. Then

$$
\begin{gathered}
\frac{\partial C_{\mathrm{JT}}^{\prime}}{\partial P_{c}}=-\frac{\mu}{P_{c}{ }^{2}}\left(\frac{(2-m) r_{v} P_{v} q}{2}+C_{L b}\right) \\
\frac{\partial^{2} C_{\mathrm{JT}}^{\prime}}{\partial P_{c}{ }^{2}}=\frac{\mu}{P_{c}{ }^{3}}\left[(2-m) r_{v} P_{v} q+2 C_{L b}\right]>0 .
\end{gathered}
$$

Therefore, there is the optimal productivity. Let (35) equal zero; there will be $C_{L b}=(m-2) r_{v} P_{v} q / 2$.

So the vendor's optimal productivity with controllable lead time has the following properties.

Corollary 4. When there is $C_{L b}=(m-2) r_{v} P_{v} q / 2$, the vendor's productivity will have an optimal value with controllable lead time.

\section{Conclusions}

Lead time is becoming increasingly important for industry, especially for fashion supply chains, which has appealed more and more to researchers' and businessmen's attention. The length of lead time represents speed and service level.
In this paper, lead time is controllable and the compressing cost of lead time follows exponential function, the assumption of which is chosen reasonably, creatively, and realistically. In this paper, we consider problems of a two-echelon fashion supply chain consisting of a single-vendor and a single-buyer. On such basis, the centralized model of the fashion supply chain inventory optimization with controllable lead time and service level constraint is proposed. The solution processes to get the optimal solutions are shown. Then we consider a price discount mechanism, which is proposed to ensure both the vendor and the buyer more profit, helping the entire fashion supply chain to reach the Pareto optimality. At the same time, we also extend the compressing cost function of [22]. We assume that the controllable lead time is sensitive to productivity, and the result can help vendors to find the optimal productivity to obtain a win-win situation for both the vendor and the buyer.

At last, the results of numerical example show that assuming the unit cost of compressing lead time to follow exponential distribution is of practical significance for fashion industry. The model provides a theoretical basis for fashion industry much managers to decide the optimal order quantity, optimal production quantity, and optimal lead time with service level constraint. And the optimal order quantity and optimal production quantity both have their boundaries due to the constraint of service level, and what is more, it makes the computation easier. In the future, more efforts can be put in studying different functions of the compressing cost of the lead time.

\section{Notations}

q: $\quad$ Order quantity of the buyer

$L: \quad$ The length of lead time for the buyer

$m$ : The number of lots in which the product is delivered from the vendor to the buyer in one production cycle, a positive integer

$\alpha: \quad$ The ratio of price discount offered by the vendor, $\alpha \in[0,1]$

$\alpha^{\prime}:$ The price discount of unit reorder product

$k$ : $\quad$ Safety coefficient, $k \geq 0$

$r: \quad$ Reorder point of the buyer

$\theta: \quad$ Service level coefficient

$B(r)$ : Expected demand shortage at the end of cycle

$X: \quad$ The lead time demand, which follows a normal distribution with finite mean $\mu L$ and standard deviation $\sigma \sqrt{L}$, where $\sigma$ denotes the standard deviation of demand per unit time, $X \sim N(\mu L, \sigma \sqrt{L})$

$P_{v}: \quad$ The vendor's unit wholesale price

$P: \quad$ The buyer's unit retail price

$P_{c}: \quad$ The vendor's production capability per year $\left(P_{c}>d\right)$

$\beta$ : $\quad$ Proportion of customers willing to wait for next replenishment, $\beta \in[0,1]$

$S: \quad$ The vendor's setup cost per setup

$r_{b}$ : Buyer's holding cost rate (per monetary unit invested in inventory) per unit time

$r_{v}$ : Vendor's holding cost rate (per monetary unit invested in inventory) per unit time. 


\section{Conflict of Interests}

The authors declare that there is no conflict of interests regarding the publication of this paper.

\section{Acknowledgments}

This work was supported by the National Natural Science Foundation of China (nos. 71102174 and 71372019), Beijing Higher Education Young Elite Teacher Project (no. YETP1173), Specialized Research Fund for Doctoral Program of Higher Education of China (no. 20111101120019), and Beijing Philosophy and Social Science Foundation of China (no. 11JGC106).

\section{References}

[1] A. Şen, "The US fashion industry: a supply chain review," International Journal of Production Economics, vol. 114, no. 2, pp. 571-593, 2008.

[2] K. Wang, Q. L. Gou, J. W. Sun, and X. H. Yue, "Coordination of a fashion and textile supply chain with demand variations," Journal of Systems Science and Systems Engineering, vol. 21, no. 4, pp. 461-479, 2012.

[3] H. J. Peng and M. H. Zhou, "Production and ordering decision in supply chain with uncertainty in two-echelon yields and demand," Journal of Systems Engineering, vol. 25, no. 5, pp. 622628, 2010 (Chinese).

[4] C. Chiang and W. C. Benton, "Sole sourcing versus dual sourcing under stochastic demands and lead times," Naval Research Logistics, vol. 41, no. 5, pp. 609-624, 1994.

[5] M. Treleven and S. Bergman Schweikhart, "A risk/benefit analysis of sourcing strategies: single vs. multiple sourcing," Journal of Operations Management, vol. 7, no. 3-4, pp. 93-114, 1988.

[6] P. Chu, K. Yang, and P. S. Chen, "Improved inventory models with service level and lead time," Computers and Operations Research, vol. 32, no. 2, pp. 285-296, 2005.

[7] M. Ben-Daya and A. Raouf, "Inventory models involving lead time as a decision variable," Journal of the Operational Research Society, vol. 45, no. 5, pp. 579-582, 1994.

[8] L. Y. Ouyang and K. S. Wu, "Mixture inventory model involving variable lead time with a service level constraint," Computers \& Operations Research, vol. 24, no. 9, pp. 875-882, 1997.

[9] M. Hariga and M. Ben-Daya, "Some stochastic inventory models with deterministic variable lead time," European Journal of Operational Research, vol. 113, no. 1, pp. 42-51, 1999.

[10] M. Ben-Daya and M. Hariga, "Lead-time reduction in a stochastic inventory system with learning consideration," International Journal of Production Research, vol. 41, no. 3, pp. 571-579, 2003.

[11] P. Chu, K. Yang, and P. S. Chen, "Improved inventory models with service level and lead time," Computers \& Operations Research, vol. 32, no. 2, pp. 285-296, 2005.

[12] G. Li, M. Q. Liu, Z. H. Wang, and B. Z. Peng, "Supply coordination based on bonus policy in assembly under uncertain delivery time," Chinese Journal of Mechanical Engineering, vol. 26, no. 2, pp. 293-303, 2013.

[13] G. Li, M. Liu, X. Guan, and Z. Huang, "Collaborative policy of the supply-hub for assemble-to-order systems with delivery uncertainty," Discrete Dynamics in Nature and Society, vol. 2014, Article ID 625812, 10 pages, 2014.

[14] C. J. Liao and C. H. Shyu, "An analytical determination of lead time with normal demand," International Journal of Operations Production Management, vol. 11, pp. 72-78, 1991.

[15] L. Y. Ouyang, N. C. Yeh, and K. S. Wu, "Mixture inventory model with backorders and lost sales for variable lead time," Journal of the Operational Research Society, vol. 47, no. 6, pp. 829-832, 1996.

[16] I. Moon and S. Choi, "A note on lead time and distributional assumptions in continuous review inventory models," Computers \& Operations Research, vol. 25, no. 11, pp. 1007-1012, 1998.

[17] S. P. Lan, P. Chu, K. J. Chung, W. J. Wan, and R. Lo, "A simple method to locate the optimal solution of the inventory model with variable lead time," Computers \& Operations Research, vol. 26, no. 6, pp. 599-605, 1999.

[18] L. Y. Ouyang, B. R. Chuang, and P. H. Chang, "Lead time reduction models with defective items subject to a service level constraint," Journal of Information \& Optimization Sciences, vol. 23, no. 1, pp. 133-146, 2002.

[19] L. Y. Ouyang and K. S. Wu, "A minimax distribution free procedure for mixed inventory model with variable lead time," International Journal of Production Economics, vol. 56-57, pp. 511-516, 1998.

[20] L. Y. Ouyang, K. S. Wu, and C. H. Ho, “The single-vendor singlebuyer integrated inventory problem with quality improvement and lead time reduction-minimax distribution-free approach," Asia-Pacific Journal of Operational Research, vol. 23, no. 3, pp. 407-424, 2006.

[21] J. K. Jha and K. Shanker, "Single-vendor multi-buyer integrated production-inventory model with controllable lead time and service level constraints," Applied Mathematical Modelling, vol. 37, no. 4, pp. 1753-1767, 2013.

[22] G. Li, X. Guan, M. Q. Liu, and M. Y. Shan, "Two-echelon inventory model with service level constraint and controllable lead time sensitive to order quantity," Journal of Central South University, vol. 20, no. 11, pp. 3324-3333, 2013.

[23] L. Ouyang, K. Wu, and C. Ho, "Integrated vendor-buyer cooperative models with stochastic demand in controllable lead time," International Journal of Production Economics, vol. 92, no. 3, pp. 255-266, 2004.

[24] W. Lee, J. Wu, and C. Lei, "Computational algorithmic procedure for optimal inventory policy involving ordering cost reduction and back-order discounts when lead time demand is controllable," Applied Mathematics and Computation, vol. 189, no. 1, pp. 186-200, 2007.

[25] B. Rho and Y. Yu, "A comparative study on the structural relationships of manufacturing practices, lead time and productivity in Japan and Korea," Journal of Operations Management, vol. 16, no. 2-3, pp. 257-270, 1998.

[26] M. S. Sajadieh and M. R. Akbari Jokar, "An integrated vendorbuyer cooperative model under stochastic supply lead-time," International Journal of Advanced Manufacturing Technology, vol. 41, no. 9-10, pp. 1043-1050, 2009.

[27] C. H. Chiu, T. M. Choi, Y. J. Li, and L. Xu, "Service competition and service war: a game theoretic analysis," Service ScienceINFORMS Journal, vol. 6, pp. 63-76, 2014.

[28] G. Hadley and T. Whitin, Analysis of Inventory Systems, Prentice Hall, Englewood Cliffs, NJ, USA, 1963. 
[29] H. Shin and W. C. Benton, "A quantity discount approach to supply chain coordination," European Journal of Operational Research, vol. 180, no. 2, pp. 601-616, 2007.

[30] J. Hu and C. L. Munson, "Incorporating quantity discounts and their inventory impacts into the centralized purchasing decision," European Journal of Operational Research, vol. 201, no. 2, pp. 581-592, 2010. 


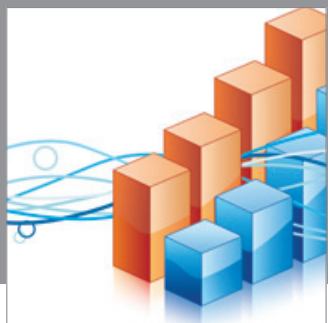

Advances in

Operations Research

mansans

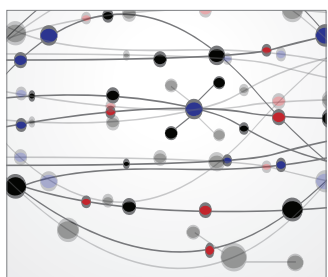

The Scientific World Journal
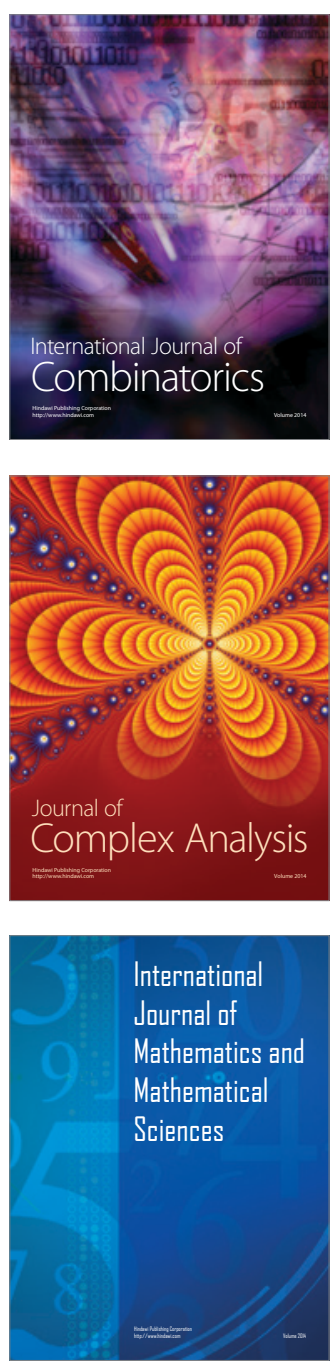
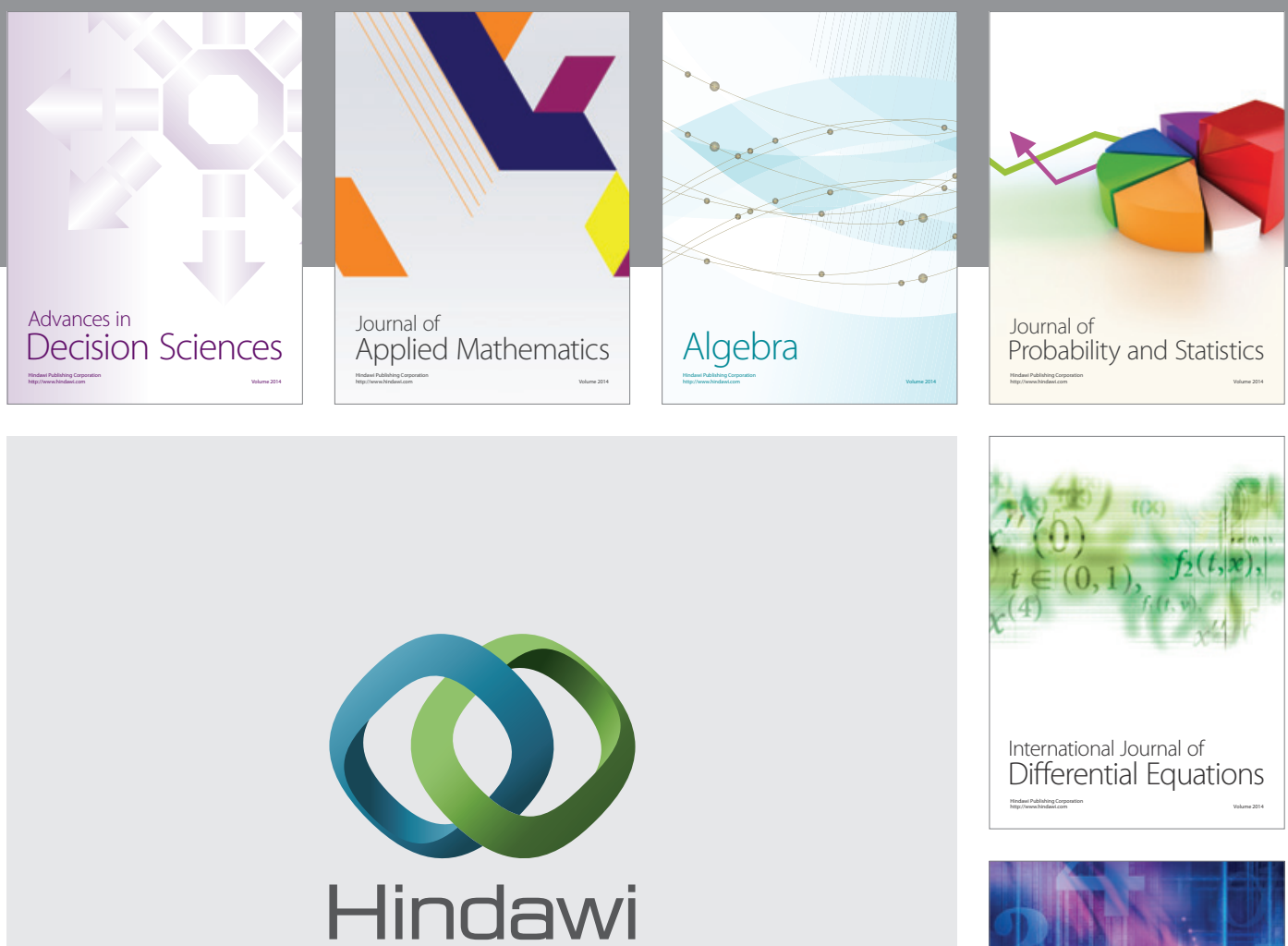

Submit your manuscripts at http://www.hindawi.com
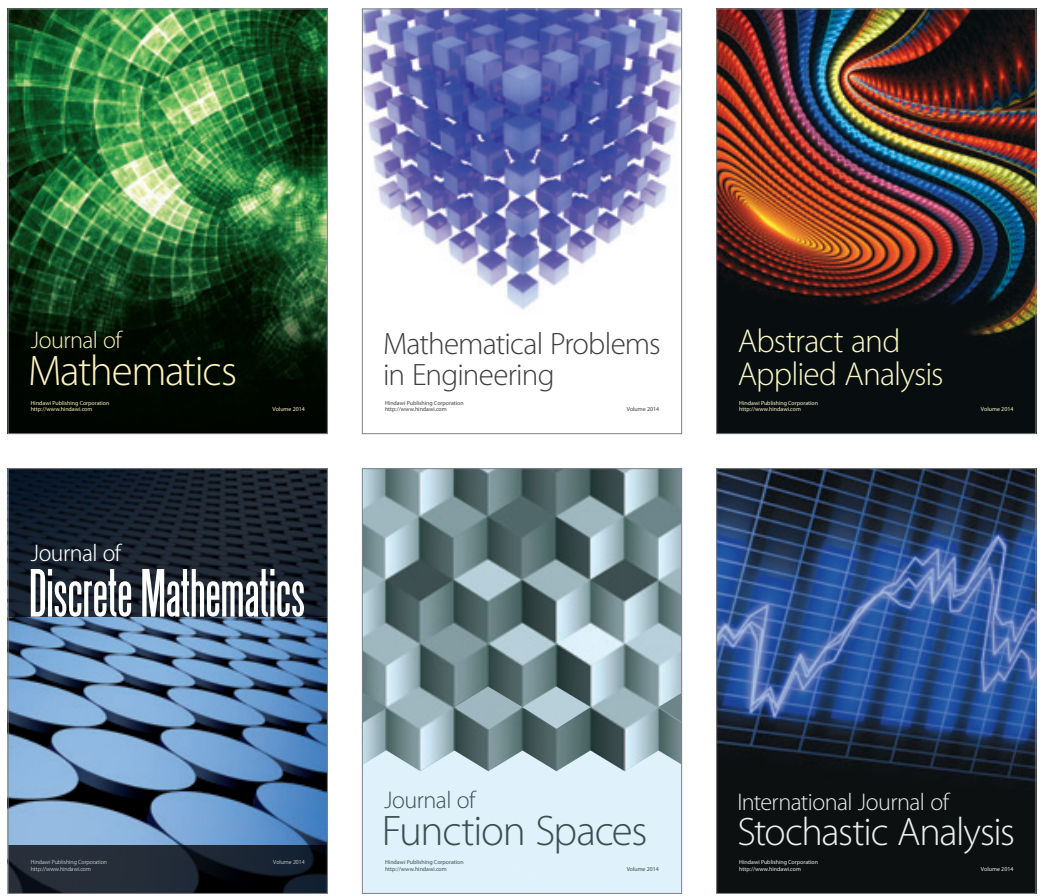

Journal of

Function Spaces

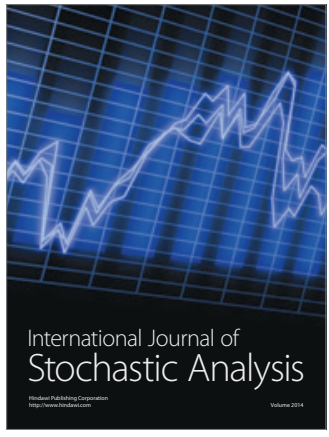

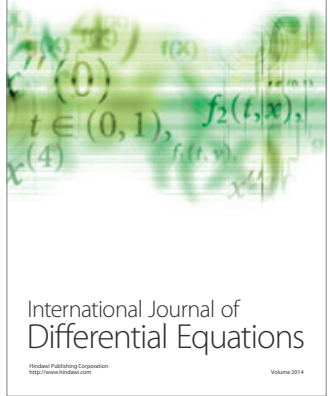
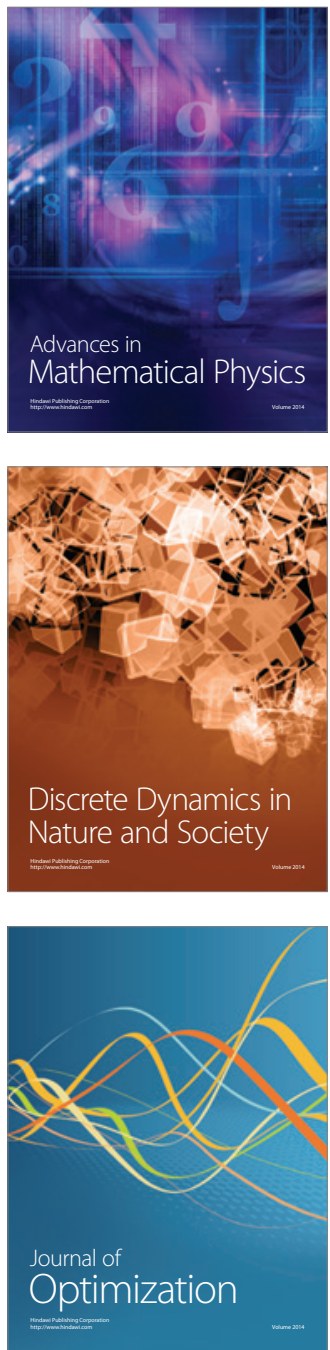